Published in final edited form as:

Nano Lett. 2020 April 08; 20(4): 2799-2805. doi:10.1021/acs.nanolett.0c00484.

\title{
Programmable Assembly of Iron Oxide Nanoparticles Using DNA Origami
}

\author{
Travis A. Meyer, \\ Wallace H. Coulter Department of Biomedical Engineering, Georgia Institute of Technology and \\ Emory University, Atlanta, Georgia 30322, United States \\ Chuan Zhang, \\ School of Chemistry and Chemical Engineering, State Key Laboratory of Metal Matrix \\ Composites, Shanghai Key Laboratory of Electrical Insulation and Thermal Ageing, Shanghai \\ Jiao Tong University, Shanghai 200240, China;
}

\section{Gang Bao, \\ Department of Bioengineering, Rice University, Houston, Texas 77005, United States;}

\section{Yonggang Ke}

Wallace H. Coulter Department of Biomedical Engineering, Georgia Institute of Technology and Emory University, Atlanta, Georgia 30322, United States;

\section{Abstract \\ Magnetic iron oxide nanoparticles (IONPs) have received significant interest for the use in biomedical applications. The assembly of IONPs into larger superstructures has been used to modify the properties and functionality of these particles. For example, the clustering of IONPs can lead to improvements in MRI contrast generation, changes in heat generation during magnetic fluid hyperthermia, and alterations to pharmacokinetics and biodistribution. Nevertheless, the IONP clustering leads to significant heterogeneity in the assembly. Here, we demonstrate a method for using DNA origami to precisely control the number and positions of IONPs. We also showed how this technique can be used to module the functionality of IONP clusters by showing how MRI contrast generation efficiency can be tuned by altering the number and spacing of IONPs. Finally, we show that these property changes can be dynamically regulated, demonstrating the possibility for this technology to be used in biosensing applications.}

\section{Graphical Abstract}

\footnotetext{
Corresponding Author: yonggang.ke@emory.edu. Author Contributions

T.A.M. and Y.K. conceived the project. T.A.M. collected experimental data and performed data analysis. The manuscript was written through contributions of all authors. All authors have given approval to the final version of the manuscript.

Supporting Information

The Supporting Information is available free of charge at https://pubs.acs.org/doi/10.1021/acs.nanolett.0c00484.

Experimental methods, $16 \mathrm{HB}$ and IONP synthesis, representative TEM images, additional figures, DNA sequences used, caDNAno design file (PDF)

The authors declare no competing financial interest.
} 


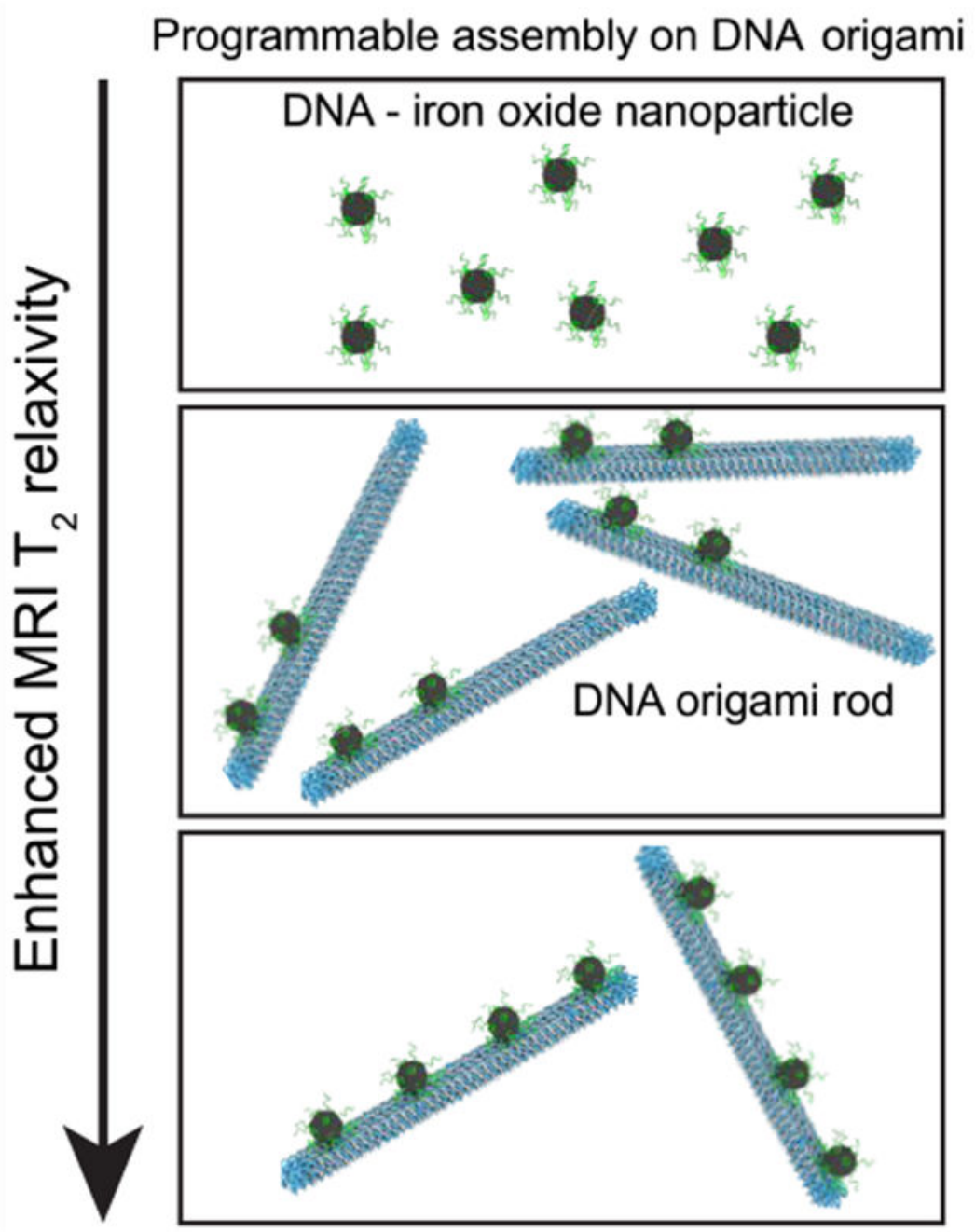

\section{Keywords}

DNA Origami; Iron Oxide Nanoparticles; Self-Assembly; Magnetic Resonance Imaging Contrast

The three-dimensional organization of nanoparticle systems play a critical role in determining the emerging functional properties and subsequent applications of these nanomaterials. Magnetic iron oxide nanoparticles (IONPs) have received significant interest for the use in biomedical applications due to their unique magnetic properties, high biocompatibility, and ease of synthesis. ${ }^{1}$ IONPs have been developed as MRI contrast agents, mediators of hyperthermia therapies, and magnetically guided vectors for drug and gene delivery. The assembly of IONPs into larger superstructures has been used to modify the emerging properties and functionality of these particles. The most conventional method for IONP assembly is the clustering of IONPs, which leads to a substantial increase in the $\mathrm{T}_{2}$ relaxivity of the particles, improving their activity as MRI contrast agents, ${ }^{2-5}$ and also been shown to modulate the specific absorption rate (SAR) and alter the efficiency of heat generation during magnetic fluid hyperthermia. ${ }^{6,7}$ Finally, the assembly of IONPs into 
anisotropic structures has been shown to improve the pharmacokinetic profile and modify the in vivo fate of the nanoparticles. ${ }^{8}$ Previous attempts to direct the assembly of IONPs have primarily involved hydrophobic interaction-driven clustering of particles inside amphiphilic polymer shells, $, 3,5$ though encapsulation inside other matrixes such as silica have also been used. ${ }^{9}$ Anisotropic IONP assemblies have also been developed using both polymer encapsulation ${ }^{8}$ as well as directed assembly using an anisotropic scaffold, such as viral proteins ${ }^{10}$ or DNA. ${ }^{11,12}$ Further improvement in the ability to control the spatial organization of IONPs within these assemblies, such as particle number, particle spacing, and overall geometry, is needed in order to further understand and develop IONP assemblies for biomedical applications.

Structural DNA nanotechnology, particularly DNA origami, has emerged as a powerful approach for the programming the self-assembly of custom-shaped one-, two-, and threedimensional nanostructures. ${ }^{13,14}$ These structures can interface in a versatile fashion with a number of other nanoscale components to direct the arrangement and interactions between guest species. DNA origami has been used to study interactions between gold nanoparticles, 15-18 organic fluorophores, ${ }^{19,20}$ quantum dots, ${ }^{21,22}$ and proteins. ${ }^{23-25}$ As such, DNA origami stands as an excellent platform for constructing well-ordered, anisotropic assemblies of IONPs with a high degree of control and precision over the spatial organization of the particles. In this work, we demonstrate a method for using DNA origami to precisely control the number and position of IONPs within an anisotropic assembly. We then show how this technique can be used to module the functionality of IONP clusters by demonstrating how MRI contrast generation efficiency can be tuned by altering the number and spacing of IONPs. Finally, we show that these property changes can be dynamically regulated, demonstrating the possibility for this technology to be used in biosensing applications.

We used a DNA origami rod consisting of 16 double helices arranged on a $4 \times 4$ square lattice (16 Helix Bundle, or 16HB) with dimensions $\sim 10 \mathrm{~nm} \times 10 \mathrm{~nm} \times 130 \mathrm{~nm}$ (Figure 1A). Additionally, four strands containing poly-A extensions were added to one end of the $16 \mathrm{HB}$ to facilitate binding to polyT coated polymeric beads for use in purification. The $16 \mathrm{HB}$ was assembled by annealing a mixture of staple strands with a long, single-stranded scaffold strand (m13 bacteriophage DNA) and agarose gel electrophoresis (AGE) and transmission electron microscopy (TEM) were used to verify successful assembly of the DNA origami rod in high yield with the expected dimensions (Supplementary Figure S1). In order to attach IONPs to the 16HB rod, we utilized complementary "capture DNA"-"cargo DNA" hybridization. Specific staple strands were modified with identical "capture" single-stranded (ss) DNA extensions, which could then hybridize to complementary "cargo" ssDNA conjugated to the surface of IONPs. A total of 36 staple strands amenable to modification with the capture sequence are located on each face of the 16HB (144 positions for all four faces). We divided these 36 strands into 12 groups of 3 strands each, to produce a total of 12 binding sites along each face of the DNA origami rod (Figure 1B). The precise location of individual IONPs could be controlled by selectively choosing the staple strands at a specific binding site to be extended with the capture sequence. We chose to include three strands at each binding site due to observations by ourselves and others ${ }^{26}$ that the incorporation of multiple capture sequences improves cargo binding yields, likely due to improved affinity 
through cooperative binding and capture site redundancy to account for defects in staple strand incorporation during assembly.

The DNA-coated IONPs was synthesized with the "Programmable Atomic Equivalent" technique developed previously. ${ }^{27}$ Commercially available hydrophobic $15 \mathrm{~nm}$ IONPs were coated with an azide-modified amphiphilic polymer poly(maleic anhydride-alt-octadecene) (PMAO) and then conjugated to ssDNA modified with the strained alkyne dibenzocyclooctyne (DBCO) (Supplementary Figure S2) using a strain-promoted azidoalkyne cycloaddition (SpAAC) reaction. Successful DNA conjugation was verified by agarose gel electrophoresis and dynamic light scattering (Supplementary Figure S3). Additional synthetic strategies using commercially available polymer-coated IONPs were investigated but produced IONP-DNA conjugates of lower quality than the method outlined above (Supplementary Figure S4)

Attachment of DNA-IONPs to the 16HB was verified using agarose gel electrophoresis and TEM imaging of gel-purified structures. Statistical analysis of TEM images was used to quantify the efficiency of binding by counting the number of 16HB with the correct number of bound IONPS (Supplementary Figure S5). We first sought to identify conditions which would lead to optimized binding yields using a 16HB with three evenly spaced binding sites (Supplementary Figure S6). We found that the binding reaction is highly robust over the conditions tested, with maximum binding yields of $>70 \%$ obtained in as little as 15 min over a range of temperatures and IONP/16HB ratios. We found that yields increased with increasing magnesium concentrations, which matches observations using other systems ${ }^{26}$ and is likely due to a reduction in the electrostatic repulsion between the 16HB and DNAcoated IONPs with increasing cation concentration.

Next, we sought to demonstrate precise control over the spacing of IONPs. We designed $16 \mathrm{HB}$ variants with two binding sites containing "capture" extensions, the first at position one and the second at positions 4-12. We found that the spacing between IONPs could be tuned between $\sim 35 \mathrm{~nm}$ and $\sim 120 \mathrm{~nm}$ with $\sim 10 \mathrm{~nm}$ spacing (Figure 1C, Supplementary Figures S7-S16). We also observed that the yield of fully assembled structures dropped as the IONPs were placed closer together, with yields sharply dropping with particle spacing of $35 \mathrm{~nm}$, and this is likely due to both steric effects and bridging of a single IONP across two neighboring binding sites and represents a lower limit on the spacing that can be obtained using $15 \mathrm{~nm}$ particles. We also tested binding using $10 \mathrm{~nm}$ IONPs and found that closer spacing could be achieved with these particles, as expected (Supplementary Figure S17).

We next sought to demonstrate control over the precise number of IONP bound to the 16HB. In this instance, we altered both the number and position of binding sites which contain the capture strand extensions. For example, by introducing binding sites at position 1, positions $1 / 6$, and positions $1 / 6 / 12$, we were able to pattern the assembly of between 1 and 3 particles with a constant $\sim 55 \mathrm{~nm}$ spacing, with electrophoretic mobility decreasing as the number of bound particles increased (Figure 2A, Supplementary Figures S18-S21). We are also able to decouple the number and spacing of bound IONPs-by introducing binding sites at position 1 , positions $1 / 4$, positions $1 / 4 / 7$, and positions $1 / 4 / 7 / 10$, we were able to attach between 1 and 4 IONPs while maintaining a constant $\sim 35 \mathrm{~nm}$ spacing (Figure 2B, Supplementary 
Figure S22-S24). Finally, we were interested in identifying the maximal number of IONPs we could attach to the 16HB. We altered the 16HB design to contain 6 alternating binding sites on each of the 4 faces of the rod, giving a theoretical maximum of 24 possible binding sites which should enable full coverage of IONPs on the entire 16HB (Figure 2C). TEM imaging revealed these "16HB-Full" structures contained high numbers of IONPs packed more closely together on multiple sides of the 16HB (Figure 2D, Supplementary Figure S25). Statistical analysis of these populations revealed 7-10 IONPs attached to each 16HB (average $8.25 \pm 0.16$ ) (Figure 2E). The decreased number of IONPS relative to binding sites is likely due to a combination of steric and electrostatic interactions as well as site bridging. Similar results for controlling the number of IONPs attached to 16HBs were also obtained using $10 \mathrm{~nm}$ particles (Supplementary Figure S26).

The yields obtained in this work are lower than those obtained with other nanoparticle systems. For example, Takabayashi et al. found $\sim 97 \%$ binding yields for $10 \mathrm{~nm}$ gold particles attached to a DNA origami rod. ${ }^{26}$ It is not entirely clear why yields with our DNAIONPs are considerably lower, one likely cause is the increased complexity of the nanoparticle due to the presence of the hydrophobic ligands and amphiphilic polymer. Another culprit could be the density of DNA grafted to the particle surface, as this technique produces particles with lower grafting densities than comparable gold particles $(17 \mathrm{pmol} /$ $\mathrm{cm}^{227} \mathrm{vs} 24 \mathrm{pmol} / \mathrm{cm}^{228}$ ). Regardless, the results presented here represent a significant improvement over previous studies that have attempted to pattern the assembly of IONPs. Rafati et al. decorated DNA origami nanotubes with IONPs, but the authors did not demonstrate any modulation over the number and spacing of the IONPs. ${ }^{12}$ Ryu et al. were able to control the number of IONPs bound to a single dsDNA using zinc-finger binding proteins. ${ }^{11}$ However, their system produced clusters with much higher polydispersity and they did not demonstrate any ability to modulate interparticle distances.

We also sought to demonstrate how the control over the organization of these IONP assemblies could be used to modulate their functional properties. For this proof-of-concept, we focused on the assembly of IONPs as a means of increasing T relaxivity $\left(r_{2}\right)$ for the generation of high-efficiency MRI contrast agents. Both experimental ${ }^{2-5,29-31}$ and theoretical ${ }^{32-35}$ studies have demonstrated that assembly of IONPs into larger clusters leads to significant enhancements in $r_{2}$ due to the increased time water protons spend diffusing through the larger magnetic field inhomogeneity (larger effective radius) produced by the IONP clusters, leading to enhanced dephasing and increased relaxation rates. ${ }^{36}$ However, there have been no experimental studies to date investigating how precise changes in particle number and spacing lead to the improvement. We used 16HB designs with between 1 and 3 binding sites with $\sim 55 \mathrm{~nm}$ spacing in order to investigate how these small changes in IONP number affect $r_{2}$. 16HB-IONP conjugates were prepared as above and were purified from excess IONPs using a polymeric-bead pulldown purification technique, modified from work by Shaw et al. ${ }^{37}$ which allowed for high yield recovery of milliliter quantity of IONP-bound 16HBs (Supplementary Figure S27). Because $T_{2}$ values are dependent upon the concentration of IONPs, we developed a concentration-independent assay for analyzing changes in $T_{2}$ induced by DNA origami-directed assembly. Heating 16HBIONP samples to high temperatures denatures the underlying DNA origami support, destroying the assembly and releasing IONPs as free particles (Supplementary Figure S28). By measuring $\mathrm{T}_{2}$ values 
before and after heating, we were able to normalize these measurements to directly assay the impact of DNA-directed assembly. Normalized $r_{2}$ values could be calculated by measuring $\mathrm{T}_{2}$ of serial dilutions of 16HB-IONP before and after heating and then calculating the difference in the slope of the linear regression; this allows us to monitor assembly effects as a percent increase in $r_{2}$ relative to free IONPs. As a comparison, the $r_{2}$ value of free DNAIONPs used in this study was $140 \mathrm{mM}^{-1} \mathrm{~s}^{-1}$.

We found variations in the number of IONPs between 1 and 3 particles attached to the 16HB led to significant alterations in $r_{2}$, with 2 bound IONPs producing an $\sim 10 \%$ increase in $r_{2}$ and 3 bound IONPs producing an $\sim 20 \%$ increase in $r_{2}$ (Figure 3A, Supplementary Figures S29-S32). This value corresponds well to theoretical simulations; a 3 particle system with $40 \mathrm{~nm}$ spacing was predicted to have an $\sim 25 \%$ increase in $r_{2} .{ }^{32}$ Further increases were obtained using assemblies with fully saturated binding ( $~ 8$ IONPs/16HB) and close packing, with a $139 \%$ increase in $r_{2}$ (Figure $3 \mathrm{~B}$ ). This enhancement matches theoretical simulations of IONPs clustered via a diffusion limited process, which predict a power law relation between IONP number $(N)$ and $r_{2}$ which corresponds to a $150 \%$ increase at $N=8 .{ }^{34}$ This enhancement is comparable to experimental results obtained using random clustering techniques; for example, we found between 100 and 200\% enhancement in our previous study, ${ }^{29}$ and other studies have shown increases of $~ 150-300 \% .{ }^{2,3,5}$ This enhancement is also significantly higher than that observed in previous studies using anisotropic assemblies, with no enhancement observed using a bacteriophage scaffold ${ }^{10}$ and a $10 \%$ increase observed using dsDNA. ${ }^{11}$ No significant difference in $r_{2}$ enhancement was observed when comparing 16HB bound with one IONP and free IONPs, providing evidence that the DNA origami support does not alter $\mathrm{T}_{2}$ relaxivity by itself (Supplementary Figure $\mathrm{S} 33$ ). We also investigated the effect of IONP spacing on $\mathrm{T}_{2}$ relaxivity using 16HB designs with 2 particles bound at distances of 50,80, and $115 \mathrm{~nm}$; we found minimal differences in $r_{2}$ enhancement using this system (Supplementary Figures S34-S37). Theoretical simulations predict only small changes in $r_{2}$ at these distances, ${ }^{32,34}$ and it is likely that any differences are obscured in a sample with a distribution of clusters with different IONP numbers, as in this study. However, we believe that effects of spacing could be observed with technical improvements in the platform: for example, using ligand exchange rather than polymeric coating to prepare DNA-IONP conjugates should lead to reduced hydrodynamic radii and closer interparticle spacing. ${ }^{38}$ Another avenue is to enrich correct 16HB-IONP conjugates using advanced purification techniques, such as fast protein liquid chromatography (FPLC), ${ }^{37,39}$ to separate $16 \mathrm{HBs}$ based on the number of bound IONP.

Finally, we were interested in using dynamic changes in the assembly of our 16HB to drive dynamic changes in $\mathrm{T}_{2}$ relaxivity as a possible mechanism for developing biosensing applications. We designed a system which enabled the tail-to-tail dimerization of $16 \mathrm{HBs}$ via introduction of strands complementary to free scaffold loops present on the end of the rods. Addition of these strands to a mixture of purified 16HB monomers leads to the formation of DNA origami rods $290 \mathrm{~nm}$ in length (Supplementary Figure S38). By adding these strands to a solution of $16 \mathrm{HB}$ bound to IONPs, the formation of dimers leads to an increase in the number of bound IONPs within each structure and thus an increase in $\mathrm{T}_{2}$ relaxivity (Figure 3C). We assembled 16HB structures with attached IONPs and added dimerization strands; after $24 \mathrm{~h}$, we observed a shift in AGE mobility consistent with 16HB dimerization and 
TEM imaging confirmed the formation of 16HB-dimers containing 4 bound IONPs (Supplementary Figure S39). To monitor the effect of dynamic dimer assembly on $\mathrm{T}_{2}$ relaxivity, we prepared two identical solutions of $16 \mathrm{HB} 2+\mathrm{IONP}$ and measured the $\mathrm{T}_{2}$ relaxation rate $\left(R_{2}\right)$ for each sample. We then added dimerization strands to one of these solutions and continued to measure $R_{2}$ values in both solutions 1,3 , and $24 \mathrm{~h}$ after dimerization strand addition. We found that $R_{2}$ increases over time for the sample with dimerization strands when normalized to the control sample, with a maximum enhancement of $\sim 13 \%$ after $24 \mathrm{~h}$ (Figure 3D, Supplementary Figure S40). After heating of the samples, the $R_{2}$ values returned to identical values, indicating that $R_{2}$ increases were due to dimerization-induced increases in the number of IONPs.

In conclusion, we have developed a novel method for the synthesis of well-ordered, anisotropic assemblies of IONPs using a DNA origami rod. With this system, we demonstrated unprecedented control over the number and spacing of IONPs within the assembly by altering the position of binding sites along the DNA origami rod, with between 35 and $120 \mathrm{~nm}$ interparticle spacing and up to 4 particles attached along one face. A maximum assembly size of $8-9$ particles was achieved by saturating the surface of the $16 \mathrm{HB}$ with binding sites and represents the upper-limit on assembly size using this system. However, the use of alternative DNA nanostructure designs, such as wireframe structures 40 or large assemblies from DNA bricks, ${ }^{41}$ offer the opportunity to further increase the number of bound IONPs and thus potentially the $\mathrm{T}_{2}$ relaxation enhancement. Additionally, we were able to demonstrate functional impacts of IONP assembly, with increasing numbers of IONPs leading to increases in $\mathrm{T}_{2}$ relaxivity in good agreement with theoretical predictions. Finally, we found that dynamic assembly of DNA origami dimers could be used to drive dynamic changes in $T_{2}$ relaxivity. This technique can be used to direct the assembly of complex arrangements of IONPs to facilitate further studies into the relationship between IONP organization and $\mathrm{T}_{2}$ relaxivity enhancement, as well as to study other functional effects, such as magnetic fluid hyperthermia and magnetic field-guided in vivo behavior. Furthermore, the incorporation of dynamic and/or stimuli responsive DNA elements offer the possibility for engineering in vitro and in vivo biosensing assays using combinations of DNA origami structures and IONPs. We envision this strategy representing a complementary technique to previous work on crude assembly of larger, polydisperse IONP clusters, providing additional detailed information on the mechanisms of relaxivity enhancement which can inform the design of future IONP clusters. Finally, the incorporation of IONPs into DNA nanostructure facilitates the use of MRI as a means of tracking the in vivo fate of this novel class of nanoparticles.

\section{Supplementary Material}

Refer to Web version on PubMed Central for supplementary material.

\section{ACKNOWLEDGMENTS}

We would like to acknowledge the support of the Robert P. Apkarian Integrated Electron Microscopy Core at Emory University for assistance with TEM imaging. We also thank Chenwei Wu for providing $10 \mathrm{~nm}$ DNA-IONPs and Dr. Hui Mao for helpful discussions related to IONPs and MRI contrast enhancement. 
Funding

This work was supported in part by the Cancer Prevention and Research Institute of Texas (CPRIT) (Grant RR14008 to G.B.) and an NSF Graduate Research Fellowship Award (Grant DGE-1148903 to T.M.). Y.K. acknowledges NIH Grant R21AI135753.

\section{REFERENCES}

(1). Gupta AK; Gupta M Synthesis and surface engineering of iron oxide nanoparticles for biomedical applications. Biomaterials 2005, 26 (18), 3995-4021. [PubMed: 15626447]

(2). Balasubramaniam S; Kayandan S; Lin Y-N; Kelly DF; House MJ; Woodward RC St. Pierre TG; Riffle JS; Davis RM Toward design of magnetic nanoparticle clusters stabilized by biocompatible diblock copolymers for T(2)-weighted MRI contrast. Langmuir 2014, 30 (6), 1580-1587. [PubMed: 24479874]

(3). Poselt E; Kloust H; Tromsdorf U; Janschel M; Hahn C; Masslo C; Weller H Relaxivity optimization of a PEGylated iron-oxide-based negative magnetic resonance contrast agent for T(2)-weighted spin-echo imaging. ACS Nano 2012, 6 (2), 1619-24. [PubMed: 22276942]

(4). Perez JM; Josephson L; O’Loughlin T; Hogemann D; Weissleder R Magnetic relaxation switches capable of sensing molecular interactions. Nat. Biotechnol 2002, 20 (8), 816-20. [PubMed: 12134166]

(5). Berret JF; Schonbeck N; Gazeau F; El Kharrat D; Sandre O; Vacher A; Airiau M Controlled clustering of superparamagnetic nanoparticles using block copolymers: design of new contrast agents for magnetic resonance imaging. J. Am. Chem. Soc 2006, 128 (5), 1755-61. [PubMed: 16448152]

(6). Andreu I; Natividad E; Solozábal L; Roubeau O Nano-objects for Addressing the Control of Nanoparticle Arrangement and Performance in Magnetic Hyperthermia. ACS Nano 2015, 9 (2), 1408-1419. [PubMed: 25658023]

(7). Dennis CL; Krycka KL; Borchers JA; Desautels RD; van Lierop J; Huls NF; Jackson AJ; Gruettner C; Ivkov R Internal Magnetic Structure of Nanoparticles Dominates Time-Dependent Relaxation Processes in a Magnetic Field. Adv. Funct. Mater 2015, 25 (27), 4300-4311.

(8). Park JH; von Maltzahn G; Zhang L; Schwartz MP; Ruoslahti E; Bhatia SN; Sailor MJ Magnetic Iron Oxide Nanoworms for Tumor Targeting and Imaging. Adv. Mater 2008, 20 (9), 1630-1635. [PubMed: 21687830]

(9). Yoon TJ; Lee H; Shao H; Hilderbrand SA; Weissleder R Multicore assemblies potentiate magnetic properties of biomagnetic nanoparticles. Adv. Mater 2011, 23 (41), 4793-7. [PubMed: 21953810]

(10). Ghosh D; Lee Y; Thomas S; Kohli AG; Yun DS; Belcher AM; Kelly KA M13-templated magnetic nanoparticles for targeted in vivo imaging of prostate cancer. Nat. Nanotechnol 2012, 7 (10), 677. [PubMed: 22983492]

(11). Ryu Y; Jin Z; Lee J.-j.; Noh S.-h.; Shin T-H; Jo S-M; Choi J; Park H; Cheon J; Kim H-S SizeControlled Construction of Magnetic Nanoparticle Clusters Using DNA-Binding Zinc Finger Protein. Angew. Chem., Int. Ed 2015, 54 (3), 923-926.

(12). Rafati A; Zarrabi A; Gill P Fabrication of DNA nanotubes with an array of exterior magnetic nanoparticles. Mater. Sci. Eng., C 2017, 79, 216-220.

(13). Rothemund PW Folding DNA to create nanoscale shapes and patterns. Nature 2006, 440 (7082), 297-302. [PubMed: 16541064]

(14). Wang P; Meyer TA; Pan V; Dutta PK; Ke Y The Beauty and Utility of DNA Origami. Chem. 2017, 2 (3), 359-382.

(15). Wang PF; Gaitanaros S; Lee S; Bathe M; Shih WM; Ke YG Programming Self-Assembly of DNA Origami Honeycomb Two-Dimensional Lattices and Plasmonic Metamaterials. J. Am. Chem. Soc 2016, 138 (24), 7733-7740. [PubMed: 27224641]

(16). Kuzyk A; Schreiber R; Fan ZY; Pardatscher G; Roller EM; Hogele A; Simmel FC; Govorov AO; Liedl T DNA-based self-assembly of chiral plasmonic nanostructures with tailored optical response. Nature 2012, 483 (7389), 311-314. [PubMed: 22422265] 
(17). Liu WY; Halverson J; Tian Y; Tkachenko AV; Gang O Self-organized architectures from assorted DNA-framed nanoparticles. Nat. Chem 2016, 8 (9), 867-873. [PubMed: 27554413]

(18). Urban MJ; Dutta PK; Wang PF; Duan XY; Shen XB; Ding BQ; Ke YG; Liu N Plasmonic Toroidal Metamolecules Assembled by DNA Origami. J. Am. Chem. Soc 2016, 138 (17), 54955498. [PubMed: 27082140]

(19). Stein IH; Steinhauer C; Tinnefeld P Single-Molecule Four-Color FRET Visualizes EnergyTransfer Paths on DNA Origami. J. Am. Chem. Soc 2011, 133 (12), 4193-4195. [PubMed: 21250689]

(20). Acuna GP; Moller FM; Holzmeister P; Beater S; Lalkens B; Tinnefeld P Fluorescence Enhancement at Docking Sites of DNA-Directed Self-Assembled Nanoantennas. Science 2012, 338 (6106), 506-510. [PubMed: 23112329]

(21). Ko SH; Du K; Liddle JA Quantum-Dot Fluorescence Lifetime Engineering with DNA Origami Constructs. Angew. Chem., Int. Ed 2013, 52 (4), 1193-1197.

(22). Samanta A; Zhou YD; Zou SL; Yan H; Liu Y Fluorescence Quenching of Quantum Dots by Gold Nanoparticles: A Potential Long Range Spectroscopic Ruler. Nano Lett 2014, 14 (9), 5052-5057. [PubMed: 25084363]

(23). Derr ND; Goodman BS; Jungmann R; Leschziner AE; Shih WM; Reck-Peterson SL Tug-of-war in motor protein ensembles revealed with a programmable DNA origami scaffold. Science 2012, 338 (6107), 662-5. [PubMed: 23065903]

(24). Fu J; Liu M; Liu Y; Woodbury NW; Yan H Interenzyme substrate diffusion for an enzyme cascade organized on spatially addressable DNA nanostructures. J. Am. Chem. Soc 2012, 134 (12), 5516-9. [PubMed: 22414276]

(25). Shaw A; Lundin V; Petrova E; Fordos F; Benson E; Al-Amin A; Herland A; Blokzijl A; Hogberg B; Teixeira AI Spatial control of membrane receptor function using ligand nanocalipers. Nat. Methods 2014, 11 (8), 841-6. [PubMed: 24997862]

(26). Takabayashi S; Klein WP; Onodera C; Rapp B; Flores-Estrada J; Lindau E; Snowball L; Sam JT; Padilla JE; Lee J; Knowlton WB; Graugnard E; Yurke B; Kuang W; Hughes WL High precision and high yield fabrication of dense nanoparticle arrays onto DNA origami at statistically independent binding sites. Nanoscale 2014, 6 (22), 13928-13938. [PubMed: 25311051]

(27). Zhang C; Macfarlane RJ; Young KL; Choi CHJ; Hao L; Auyeung E; Liu G; Zhou X; Mirkin CA A general approach to DNA-programmable atom equivalents. Nat. Mater 2013, 12, 741. [PubMed: 23685863]

(28). Hurst SJ; Lytton-Jean AK; Mirkin CA Maximizing DNA loading on a range of gold nanoparticle sizes. Anal. Chem 2006, 78 (24), 8313-8318. [PubMed: 17165821]

(29). Meyer TA; Quinto CA; Bao G Control of iron oxide nanoparticle clustering using dual solvent exchange. IEEE Magn. Lett 2016, 7, 1-4.

(30). Bruns OT; Ittrich H; Peldschus K; Kaul MG; Tromsdorf UI; Lauterwasser J; Nikolic MS; Mollwitz B; Merkel M; Bigall NC; Sapra S; Reimer R; Hohenberg H; Weller H; Eychmuller A; Adam G; Beisiegel U; Heeren J Real-time magnetic resonance imaging and quantification of lipoprotein metabolism in vivo using nanocrystals. Nat. Nanotechnol 2009, 4 (3), 193-201. [PubMed: 19265850]

(31). Min C; Shao H; Liong M; Yoon T-J; Weissleder R; Lee H Mechanism of Magnetic Relaxation Switching Sensing. ACS Nano 2012, 6 (8), 6821-6828. [PubMed: 22762250]

(32). Matsumoto Y; Jasanoff A T2 relaxation induced by clusters of superparamagnetic nanoparticles: Monte Carlo simulations. Magn. Reson. Imaging 2008, 26 (7), 994-8. [PubMed: 18479873]

(33). Vuong QL; Gillis P; Gossuin Y Monte Carlo simulation and theory of proton NMR transverse relaxation induced by aggregation of magnetic particles used as MRI contrast agents. J. Magn. Reson 2011, 212 (1), 139-48. [PubMed: 21807538]

(34). Brown KA; Vassiliou CC; Issadore D; Berezovsky J; Cima MJ; Westervelt RM Scaling of transverse nuclear magnetic relaxation due to magnetic nanoparticle aggregation. J. Magn. Magn. Mater 2010, 322 (20), 3122-3126. [PubMed: 20689678]

(35). Roch A; Gossuin Y; Muller RN; Gillis P Super-paramagnetic colloid suspensions: Water magnetic relaxation and clustering. J. Magn. Magn. Mater 2005, 293 (1), 532-539. 
(36). Vuong QL; Gillis P; Roch A; Gossuin Y Magnetic resonance relaxation induced by superparamagnetic particles used as contrast agents in magnetic resonance imaging: a theoretical review. WIREs Nanomedicine and Nanobiotechnology 2017, 9 (6), No. e1468.

(37). Shaw A; Benson E; Högberg B Purification of Functionalized DNA Origami Nanostructures. ACS Nano 2015, 9 (5), 4968-4975. [PubMed: 25965916]

(38). Amstad E; Gillich T; Bilecka I; Textor M; Reimhult E Ultrastable Iron Oxide Nanoparticle Colloidal Suspensions Using Dispersants with Catechol-Derived Anchor Groups. Nano Lett. 2009, 9 (12), 4042-4048. [PubMed: 19835370]

(39). Wagenbauer KF; Engelhardt FAS; Stahl E; Hechtl VK; Stömmer P; Seebacher F; Meregalli L; Ketterer P; Gerling T; Dietz H How We Make DNA Origami. ChemBioChem 2017, 18 (19), 1873-1885. [PubMed: 28714559]

(40). Zhang F; Jiang S; Wu S; Li Y; Mao C; Liu Y; Yan H Complex wireframe DNA origami nanostructures with multi-arm junction vertices. Nat. Nanotechnol 2015, 10 (9), 779. [PubMed: 26192207]

(41). Ong LL; Hanikel N; Yaghi OK; Grun C; Strauss MT; Bron P; Lai-Kee-Him J; Schueder F; Wang B; Wang P; Kishi JY; Myhrvold C; Zhu A; Jungmann R; Bellot G; Ke Y; Yin P Programmable self-assembly of three-dimensional nanostructures from 10,000 unique components. Nature 2017, 552 (7683), 72-77. [PubMed: 29219968] 
A $16 \mathrm{HB}$
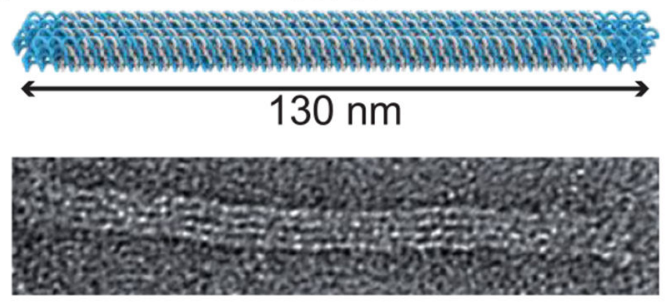

C

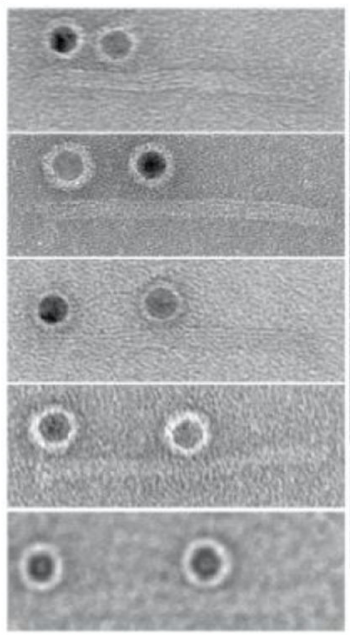

B
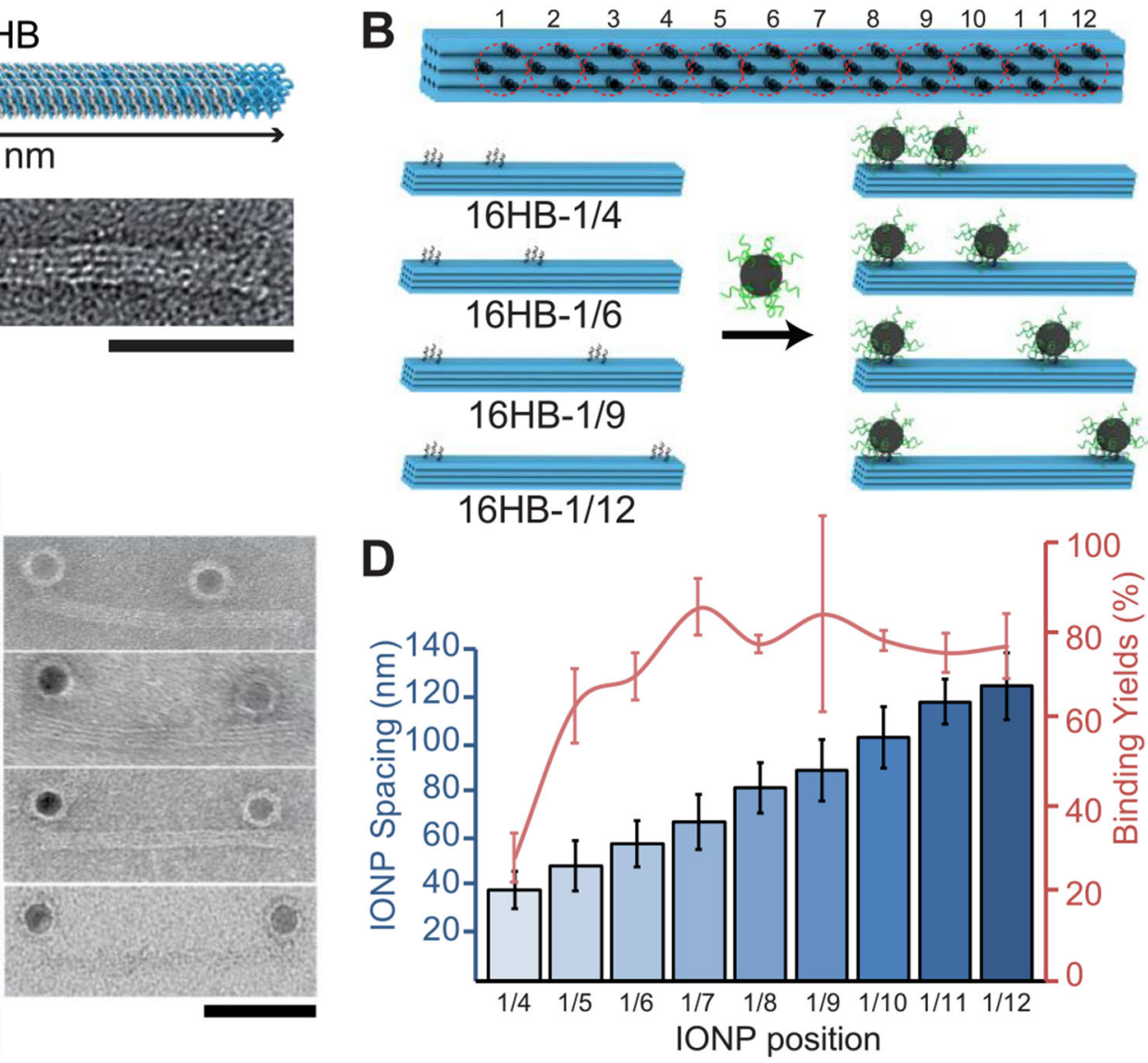

Figure 1.

Schematic of 16HB-IONP binding and control of IONP spacing. (A) Model of 16 Helix Bundle (16HB) showing scaffold (blue) and staple (gray) strands (top) and representative TEM image (bottom). (B) Schematic of 16HB showing the arrangement of "capture" DNA extensions arranged to produce 12 binding sites (top). Selective incorporation of staple strands containing extensions only at specific sites enables the placement of IONPs at discrete positions along 16HB (bottom). (C) Representative TEM images showing 16HB with 2 bound IONPs located at position 1 and positions 4-12. (D) Center-to-center interparticle spacing as a function of binding site position for 16HB's containing 2 bound IONPs (blue chart) and yields of 16HBs containing exactly 2 bound particles as a function of binding site position (red line). Results presented as mean \pm SD across two independent experiments. Sample size $N=52$ (16HB-1/4), 57(16HB-1/5), 86 (16HB-1/6), 88 (16HB-1/7), 73 (16HB-1/8), 98 (16HB-1/9), 132 (16HB-1/10), 67 (16HB-1/11), 119 (16HB-1/12). Scale bars: $50 \mathrm{~nm}$. 
A
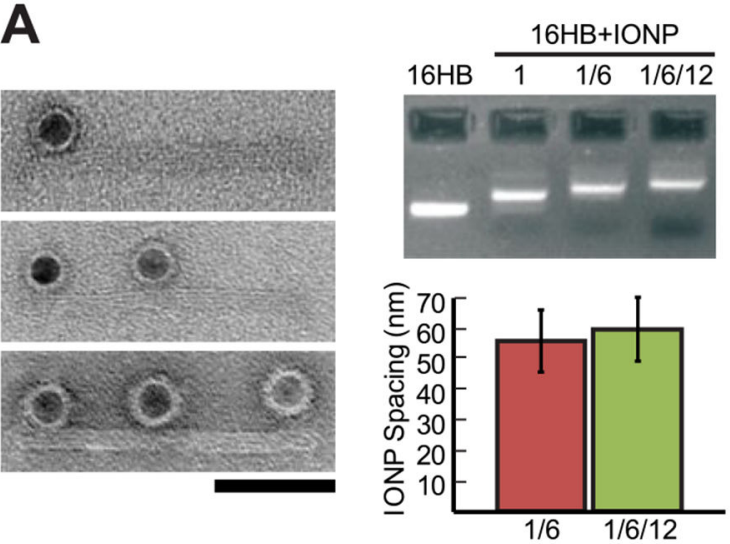

B
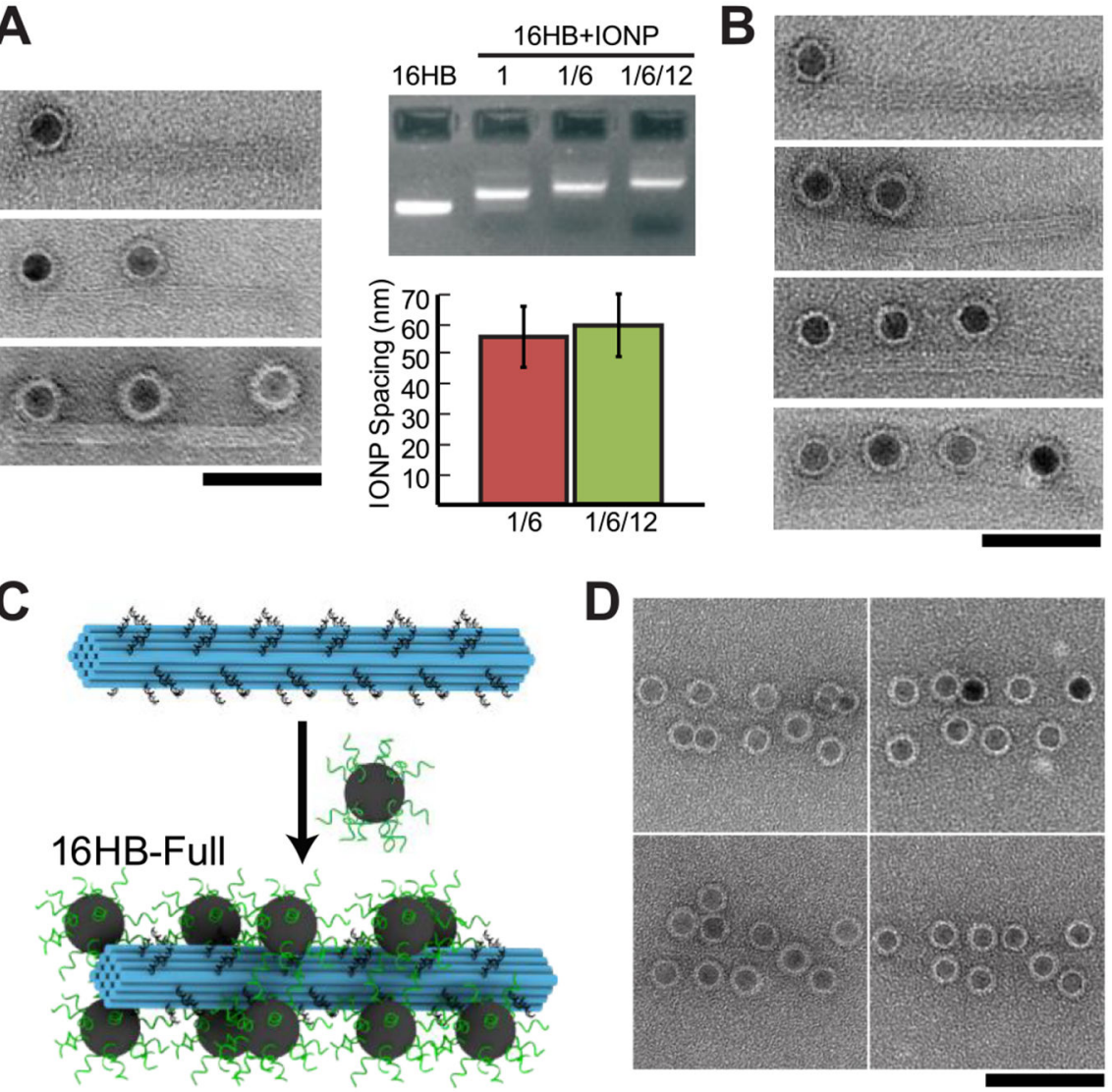

D

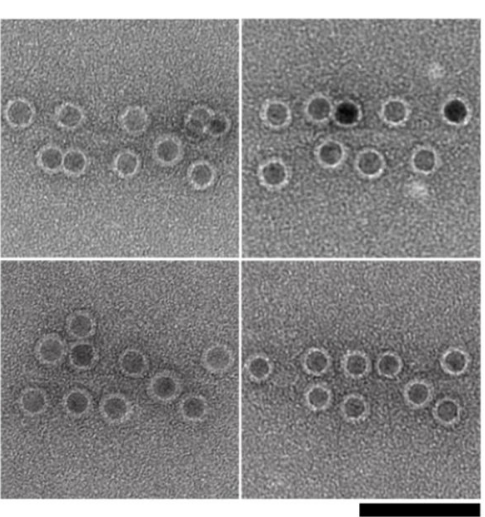

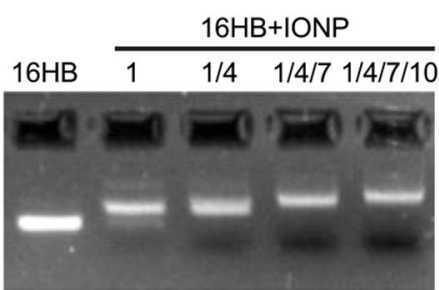

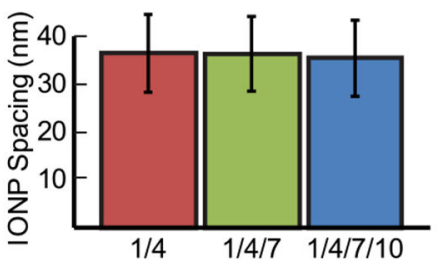

E

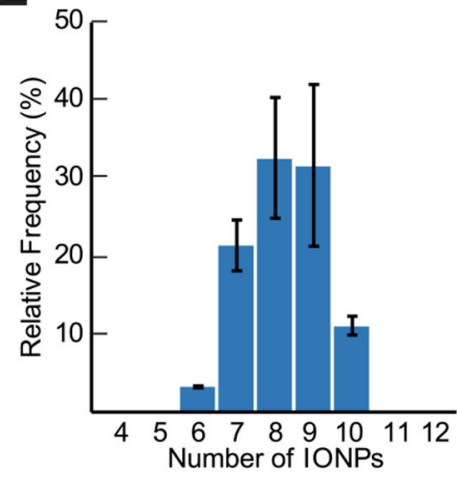

Figure 2.

Control of IONP Number. A-B) Representative TEM images (left), agarose gel electrophoresis (top right) and calculated center-to-center interparticle spacing (bottom right) of 16HB designs containing binding sites for either A) 1-3 particles at binding sites 1/6/12 with $\sim 55 \mathrm{~nm}$ spacing or B) 1-4 particles at binding sites 1/4/7/10 containing $\sim 35 \mathrm{~nm}$ spacing. C) Schematic of $16 \mathrm{HB}$ containing full coverage (of 24 binding sites across all 4 faces (16HB-Full) facilitating high density coverage of IONPs. D) Representative TEM images of 16HB-Full bound to $15 \mathrm{~nm}$ IONPs. E) Population analysis of 16HB-Full based on number of bound particles. Average: $8.25 \pm 0.16 \mathrm{IONPs} / 16 \mathrm{HB}$. Results are presented as mean \pm s.d. across two independent experiments. Sample size $N=187$ (16HB-1/6), 103 (16HB-1/6/12), 99 (16HB-1/4), 95 (16HB-1/4/7), 82 (16HB-1/4/7/10), 233 (16HB-Full) across three independent experiments. Scale bar: $50 \mathrm{~nm}$. 

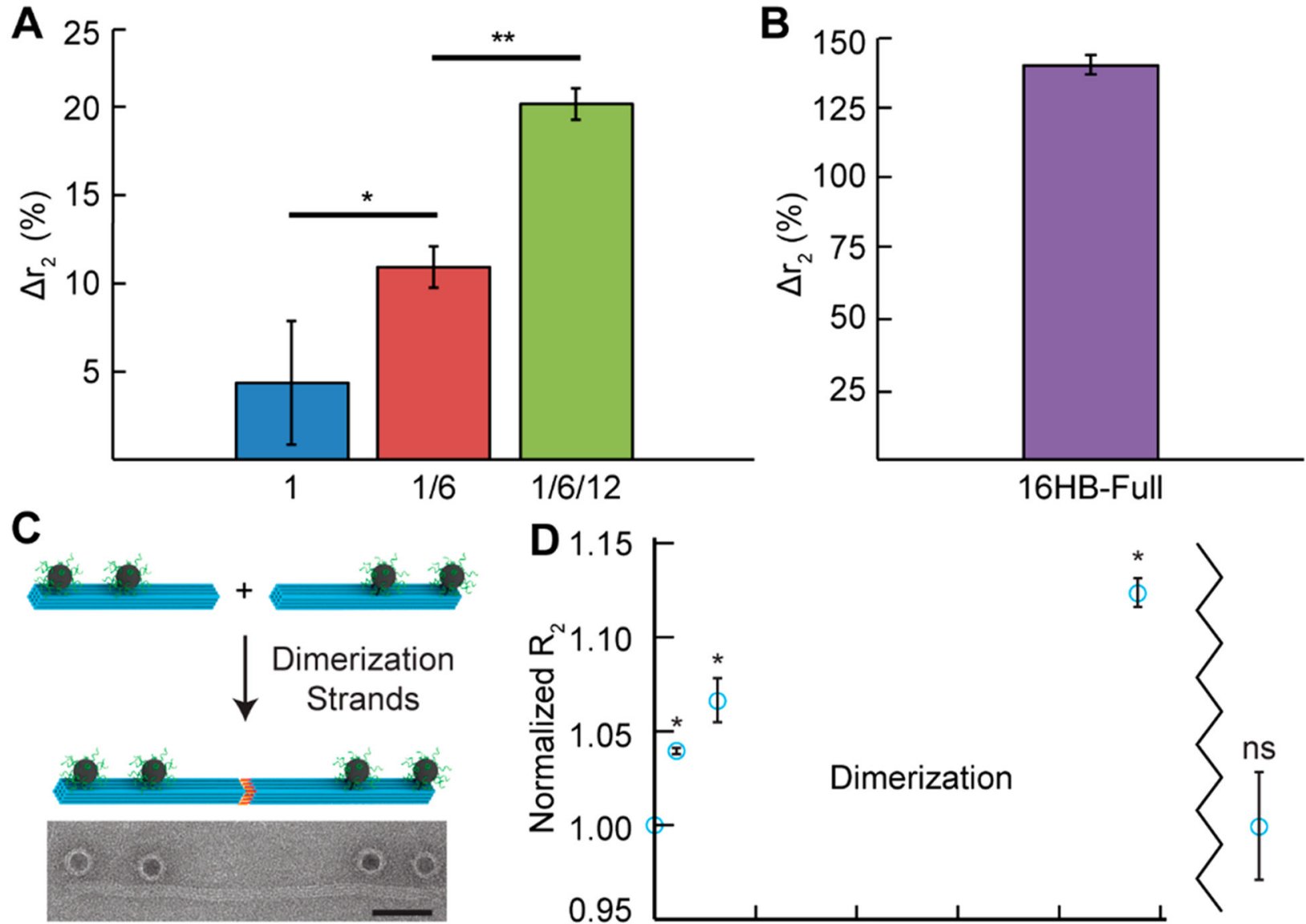

Figure 3.

Modulation of $\mathrm{T}_{2}$ relaxivity of IONPs assembled on 16HB. (A) Relative change in $\mathrm{T}_{2}$ relaxivity as a function of the number of bound particles (blue, 1; red, 2; green, 3) following heating and release of free IONPs. (B) Relative change in $\mathrm{T}_{2}$ relaxivity of $16 \mathrm{HB}-\mathrm{Full}+\mathrm{IONP}$ structures containing $\sim 8$ IONPs. (C) Schematic (top) and representative TEM image (bottom) of 16HB-IONP dimerization allowing for real-time switching of number of IONPs/ structure. (D) Relative change in $\mathrm{T}_{2}$ relaxation rate between $16 \mathrm{HB} 2(1 / 6)$-IONP samples with and without addition of dimerization strands over time, showing increase in $R_{2}$ overtime for a sample containing dimerization strands. No difference between samples was observed following heat-based denaturation of $16 \mathrm{HB}$ support and release of free IONPs. Results are presented as mean $\pm \mathrm{SD}$, three independent experiments for panels $\mathrm{A}$ and $\mathrm{B}$, two for panel D. $* P<0.05, * * P<0.0005$ Scale bar: $50 \mathrm{~nm}$. 